\title{
A note on Terai's conjecture concerning primitive Pythagorean triples
}

\author{
Maohua Le ${ }^{1}$ (D), Gökhan Soydan*2 (D) \\ ${ }^{1}$ Institute of Mathematics, Lingnan Normal College, Zhangjiang, Guangdong, 524048 China \\ ${ }^{2}$ Department of Mathematics, Bursa Uludă̆ University, Görükle Campus, 16059 Bursa, Turkey
}

\begin{abstract}
Let $f, g$ be positive integers such that $f>g, \operatorname{gcd}(f, g)=1$ and $f \not \equiv g(\bmod 2)$. In 1993, $\mathrm{N}$. Terai conjectured that the equation $x^{2}+\left(f^{2}-g^{2}\right)^{y}=\left(f^{2}+g^{2}\right)^{z}$ has only one positive integer solution $(x, y, z)=(2 f g, 2,2)$. This is a problem that has not been solved yet. In this paper, using elementary number theory methods with some known results on higher Diophantine equations, we prove that if $f=2^{r} s$ and $g=1$, where $r, s$ are positive integers satisfying $2 \nmid s, r \geq 2$ and $s<2^{r-1}$, then Terai's conjecture is true.
\end{abstract}

Mathematics Subject Classification (2020). 11D61

Keywords. polynomial-exponential Diophantine equation, generalized Ramanujan-Nagell equation, primitive Pythagorean triple

\section{Introduction}

Let $\mathbb{Z}, \mathbb{N}$ be the sets of integers and positive integers, respectively. Let $(a, b, c)$ be a primitive Pythagorean triple with $2 \mid a$. Then we have

$$
\begin{aligned}
& a=2 f g, b=f^{2}-g^{2}, c=f^{2}+g^{2} f, g \in \mathbb{N}, \\
& f>g, \operatorname{gcd}(f, g)=1, f \not \equiv g \quad(\bmod 2) .
\end{aligned}
$$

Many of the unsolved problems in number theory are related to the properties of such triples. In 1993, N. Terai [11] proposed the following conjecture about the generalized Ramanujan-Nagell equation with primitive Pythagorean triples.

Conjecture 1.1. Let $(a, b, c)$ be a primitive Pythagorean triple with $2 \mid a$. Then the equation

$$
x^{2}+b^{y}=c^{z}
$$

has only one solution $(x, y, z)=(a, 2,2)$.

This is a problem that is far from resolved. So far it has only been proved for a few special cases, mainly where $b$ or $c$ is an odd prime power (see Section 2.3 of [7]). In practical terms, Conjecture 1.1 has been verified in the following cases:

(i) (N. Terai $[11]) b \equiv 1(\bmod 4), b^{2}+1=2 c, b$ and $c$ are odd primes satisfying some conditions.

\footnotetext{
*Corresponding Author.

Email addresses: lemaohua2008@163.com (M. Le), gsoydan@uludag.edu.tr (G. Soydan)

Received: 16.09.2020; Accepted: 30.01.2021
} 
(ii) (X.-G. Chen and M.-H. Le [2]) $b \not \equiv 1(\bmod 16), b^{2}+1=2 c, b$ and $c$ are odd primes.

(iii) (M.-H. Le [5]) $b \equiv \pm 3(\bmod 8), b>8 \cdot 10^{6}, c$ is an odd prime power.

(iv) (P.-Z. Yuan [12]; P.-Z. Yuan and J.-B. Wang [13]) $b \equiv \pm 3(\bmod 8), c$ is an odd prime power.

(v) (M.-H. Le [6]; J.-Y. Hu and H. Zhang [4]) $b \equiv 7(\bmod 8), b$ or $c$ is an odd prime power.

Recently, a survey paper on the conjecture of Terai has been published by G. Soydan, M. Demirci, I.N. Cangül and A. Togbé (see [10] for the details about this conjecture).

By (1.1), (1.2) can be expressed as

$$
x^{2}+\left(f^{2}-g^{2}\right)^{y}=\left(f^{2}+g^{2}\right)^{z}, x, y, z \in \mathbb{N} .
$$

In this paper, using elemantary number theory methods with some known results on higher Diophantine equations, we deal with (1.3) in a case where neither $b$ or $c$ is a prime power. We prove the following result:

Theorem 1.2. If $f, g$ satisfy

$$
f=2^{r} s, g=1, r, s \in \mathbb{N}, 2 \nmid s, r \geq 2, s<2^{r-1},
$$

then (1.3) has only one solution $(x, y, z)=\left(2^{r+1} s, 2,2\right)$.

Thus it can be seen that if $f, g$ satisfy (1.4), then Conjecture 1.1 is true.

\section{Preliminaries}

Let $n$ be a positive integer.

Lemma 2.1 (Theorems 1.75 and 1.76 of [8]). For any complex numbers $\alpha$ and $\beta$, we have

$$
\alpha^{n}+\beta^{n}=\sum_{i=0}^{[n / 2]}(-1)^{i}\left[\begin{array}{c}
n \\
i
\end{array}\right](\alpha+\beta)^{n-2 i}(\alpha \beta)^{i} .
$$

where

$$
\left[\begin{array}{c}
n \\
i
\end{array}\right]=\frac{(n-i-1) ! n}{(n-2 i) ! i !} \in \mathbb{N}, i=0,1 \cdots,[n / 2],
$$

$[n / 2]$ is the integer part of $n / 2$.

Lemma 2.2 (The equality (2.35) of [3]). If $2 \nmid n$, then

$$
\sum_{i=0}^{(n-1) / 2}(-1)^{i}\left(\begin{array}{c}
n \\
2 i
\end{array}\right)=(-1)^{\left(n^{2}-1\right) / 8} 2^{(n-1) / 2} .
$$

Lemma 2.3 ([9]). Every solution $(X, Y, Z)$ of the equation

$$
X^{2}+Y^{2}=Z^{n}, X, Y, Z \in \mathbb{N}, \operatorname{gcd}(X, Y)=1,2 \mid Y
$$

can be expressed as

$$
\begin{aligned}
& Z=u^{2}+v^{2}, u, v \in \mathbb{N} \operatorname{gcd}(u, v)=1,2 \mid v, \\
& X+Y \sqrt{-1}=\lambda_{1}\left(u+\lambda_{2} v \sqrt{-1}\right)^{n} \lambda_{1}, \lambda_{2} \in\{1,-1\} .
\end{aligned}
$$

Lemma 2.4 ([1]). If $n \geq 4$, then the equation

$$
X^{4}+Y^{2}=Z^{n}, X, Y, Z \in \mathbb{N}, \operatorname{gcd}(X, Y)=1
$$

has no solutions.

Lemma 2.5. Let $r, s$ be positive integers satisfying $2 \nmid s$ and $s<2^{r-1}$. Then the equation

$$
2^{2 r} s^{2}+1=u^{2}+v^{2}, u, v \in \mathbb{N}, \operatorname{gcd}(u, v)=1,2^{r} \mid v
$$

has only the solution

$$
(u, v)=\left(1,2^{r} s\right)
$$


Proof. We now assume that $(u, v)$ is a solution of (2.4). Since $2^{r} \mid v$, we have $2 \nmid u$ and

$$
v=2^{r} t, t \in \mathbb{N} \text {. }
$$

Substitute (2.6) into (2.4), we get

$$
2^{2 r}\left(s^{2}-t^{2}\right)=u^{2}-1
$$

When $u=1$, by (2.7), we have $t=s$. Hence, by (2.6), we get (2.5).

When $u>1$, let $\zeta=(-1)^{(u-1) / 2}$. Then $u-\zeta$ and $u+\zeta$ are positive integers satisfying

$$
u-\zeta \equiv 0 \quad(\bmod 4), u+\zeta \equiv 2 \quad(\bmod 4) .
$$

Since $u^{2}-1=(u-\zeta)(u+\zeta)$, by $(2.7)$ and $(2.8)$, we get $u-\zeta \equiv 0\left(\bmod 2^{2 r-1}\right)$. So we have

$$
u=2^{2 r-1} \ell+\zeta, \ell \in \mathbb{N} .
$$

Substitute (2.9) into (2.7), we get

$$
s^{2}-t^{2}=\ell\left(2^{2 r-2} \ell+\zeta\right) .
$$

Therefore, by (2.10), we have

$$
s^{2}-1 \geq s^{2}-t^{2} \geq 2^{2 r-2}+\zeta \geq 2^{2 r-2}-1,
$$

whence we get $s \geq 2^{r-1}$, a contradiction. Thus, (2.4) has only the solution (2.5). The lemma is proved.

\section{Proof of Theorem 1.2}

We now assume that $(x, y, z)$ is a solution of (1.3), and $f, g$ satisfy (1.4). Then we have

$$
x^{2}+\left(2^{2 r} s^{2}-1\right)^{y}=\left(2^{2 r} s^{2}+1\right)^{z}, x, y, z \in \mathbb{N} .
$$

Since $2^{2 r} s^{2}-1$ and $2^{2 r} s^{2}+1$ are both odd, by (3.1), we get

$$
2 \mid x \text {. }
$$

Further, since $2^{2 r} s^{2}-1 \equiv-1(\bmod 4), 2^{2 r} s^{2}+1 \equiv 1(\bmod 4)$ and $x^{2} \equiv 0(\bmod 4)$ by (3.2), we get from (3.1) that

$$
2 \mid y \text {. }
$$

Also, by (3.1) and (3.3), we have $\left(2^{2 r} s^{2}+1\right)^{z}>\left(2^{2 r} s^{2}-1\right)^{y} \geq\left(2^{2 r} s^{2}-1\right)^{2}>\left(2^{2 r} s^{2}+1\right)$, whence we get

$$
z \geq 2
$$

By (3.1), (3.3) and (3.4), we have

$$
\begin{aligned}
x^{2} & =\left(2^{2 r} s^{2}+1\right)^{z}-\left(2^{2 r} s^{2}-1\right)^{y} \\
& =2^{2 r} s^{2}\left((z+y)+2^{2 r} s^{2}\left(\sum_{i=2}^{z}\left(\begin{array}{c}
z \\
i
\end{array}\right)\left(2^{2 r} s^{2}\right)^{i-2}-\sum_{j=2}^{y}(-1)^{j}\left(\begin{array}{l}
y \\
j
\end{array}\right)\left(2^{2 r} s^{2}\right)^{j-2}\right)\right) .
\end{aligned}
$$

We see from (3.5) that $2^{r} s \mid x$ and

$$
\left(\frac{x}{2^{r} s}\right)^{2} \equiv z+y \quad\left(\bmod 2^{2 r} s^{2}\right)
$$

So we have

$$
x=2^{r} s x_{1}, x_{1} \in \mathbb{N}
$$

and

$$
x_{1}^{2} \equiv z+y \quad(\bmod 4) .
$$

On the other hand, we see from (3.1), (3.2) and (3.3) that (2.2) has a solution

$$
(X, Y, Z)=\left(\left(2^{2 r} s^{2}-1\right)^{y / 2}, x, 2^{2 r} s^{2}+1\right)
$$


for $n=z$. Applying Lemma 2.3 to (3.8), we have

$$
2^{2 r} s^{2}+1=u^{2}+v^{2}, u, v \in \mathbb{N}, \operatorname{gcd}(u, v)=1,2 \mid v
$$

and

$$
\left(2^{2 r} s^{2}-1\right)^{y / 2}+x \sqrt{-1}=\lambda_{1}\left(u+\lambda_{2} v \sqrt{-1}\right)^{z}, \lambda_{1}, \lambda_{2} \in\{1,-1\} .
$$

When $2 \nmid z$, we get from (3.10) that

$$
\left(2^{2 r} s^{2}-1\right)^{y / 2}=u\left|\sum_{i=0}^{(z-1) / 2}(-1)^{i}\left(\begin{array}{c}
z \\
2 i
\end{array}\right) u^{z-2 i-1} v^{2 i}\right|
$$

and

$$
x=v\left|\sum_{i=0}^{(z-1) / 2}(-1)^{i}\left(\begin{array}{c}
z \\
2 i+1
\end{array}\right) u^{z-2 i-1} v^{2 i}\right| .
$$

Since $2 \mid v$ and $2 \nmid z$, we have $2 \nmid u$ and

$$
2 \nmid \sum_{i=0}^{(z-1) / 2}(-1)^{i}\left(\begin{array}{c}
z \\
2 i+1
\end{array}\right) u^{z-2 i-1} v^{2 i} .
$$

Hence, by (3.6), (3.12) and (3.13), we get

$$
2^{r} \mid v
$$

Therefore, by Lemma 2.5, we deduce from (3.9) and (3.14) that $u$ and $v$ satisfy (2.5). Substitute (2.5) into (3.11), we have

$$
\left(2^{2 r} s^{2}-1\right)^{y / 2}=\left|\sum_{i=0}^{(z-1) / 2}(-1)^{i}\left(\begin{array}{c}
z \\
2 i
\end{array}\right)\left(2^{2 r} s^{2}\right)^{i}\right| .
$$

Since $y / 2 \geq 1$ and $2^{2 r} s^{2} \equiv 1\left(\bmod 2^{2 r} s^{2}-1\right)$, by $(3.15)$, we get

$$
\sum_{i=0}^{(z-1) / 2}(-1)^{i}\left(\begin{array}{c}
z \\
2 i
\end{array}\right) \equiv 0 \quad\left(\bmod 2^{2 r} s^{2}-1\right) .
$$

Further, by Lemma 2.2, we obtain from (3.16) that

$$
2^{(z-1) / 2} \equiv 0 \quad\left(\bmod 2^{2 r} s^{2}-1\right) .
$$

But, since $2^{2 r} s^{2}-1$ is an odd integer with $2^{2 r} s^{2}-1>1$, (3.17) is impossible. So, we have

$$
2 \mid z \text {. }
$$

We see from (3.1), (3.2),(3.3) and (3.18) that (2.2) has a solution

$$
(X, Y, Z)=\left(\left(2^{2 r} s^{2}-1\right)^{y / 2}, x,\left(2^{2 r} s^{2}+1\right)^{z / 2}\right)
$$

for $n=2$. Applying Lemma 2.3 to (3.19), we get

$$
\begin{aligned}
& \left(2^{2 r} s^{2}-1\right)^{y / 2}=U^{2}-V^{2}, x=2 U V,\left(2^{2 r} s^{2}+1\right)^{z / 2}=U^{2}+V^{2}, \\
& U, V \in \mathbb{N}, U>V \operatorname{gcd}(U, V)=1, U \not \equiv V \quad(\bmod 2) .
\end{aligned}
$$

Further, by (3.20), we have

$$
\left(2^{2 r} s^{2}-1\right)^{y}=\left(U^{2}-V^{2}\right)^{2} \geq(U+V)^{2}>U^{2}+V^{2}=\left(2^{2 r} s^{2}+1\right)^{z / 2}>\left(2^{2 r} s^{2}-1\right)^{z / 2},
$$

whence we get

$$
y>\frac{z}{2}
$$

By (3.3), (3.7) and (3.18), we have

$$
2 \mid x_{1}
$$


Hence, by (3.7) and (3.22), we get

$$
z+y \equiv 0 \quad(\bmod 4)
$$

If $4 \mid z$, then from (3.23) we get $4 \mid y$. It follows from (3.1) that (2.3) has a solution $(X, Y, Z)=\left(\left(2^{2 r} s^{2}-1\right)^{y / 4}, x, 2^{2 r} s^{2}+1\right)$ for $n=z$. But, since $4 \mid z$ and $z \geq 4$, by Lemma 2.4 , it is impossible. So we have

$$
z \equiv y \equiv 2 \quad(\bmod 4) .
$$

Since $2 \mid z$, by (3.10), we have

$$
\left(2^{2 r} s^{2}-1\right)^{y / 2}=\left|\sum_{i=0}^{z / 2}(-1)^{i}\left(\begin{array}{c}
z \\
2 i
\end{array}\right) u^{z-2 i} v^{2 i}\right|
$$

and

$$
x=u v\left|\sum_{i=0}^{z / 2-1}(-1)^{i}\left(\begin{array}{c}
z \\
2 i+1
\end{array}\right) u^{z-2 i-2} v^{2 i}\right| .
$$

Since $2 \nmid u, 2 \mid v$ and $2 \| z$ by (3.24), we have

$$
2 \| \sum_{i=0}^{z / 2-1}(-1)^{i}\left(\begin{array}{c}
z \\
2 i+1
\end{array}\right) u^{z-2 i-2} v^{2 i} .
$$

Hence, by (3.6), (3.22), (3.26) and (3.27), we get (3.14). Therefore, by Lemma 2.5, we see from (3.9) and (3.14) that $u$ and $v$ satisfy (2.5).

Substitute (2.5) into (3.25), we have

$$
\left(2^{2 r} s^{2}-1\right)^{y / 2}=\left|\sum_{i=0}^{z / 2}(-1)^{i}\left(\begin{array}{c}
z \\
2 i
\end{array}\right)\left(2^{2 r} s^{2}\right)^{i}\right| .
$$

Let

$$
\theta=1+2^{r} s \sqrt{-1}, \bar{\theta}=1-2^{r} s \sqrt{-1} .
$$

By (3.28) and (3.29), we get

$$
\left(2^{2 r} s^{2}-1\right)^{y / 2}=\frac{1}{2}\left|\theta^{z}+\bar{\theta}^{z}\right|
$$

Further let

$$
\alpha=\theta^{2}, \beta=\bar{\theta}^{2}
$$

By (3.29) and (3.31), we have

$$
\alpha+\beta=-2\left(2^{2 r} s^{2}-1\right), \alpha \beta=\left(2^{2 r} s^{2}+1\right)^{2} .
$$

Since $z / 2$ is odd by (3.24), applying Lemma 2.1 to (3.30), we get from (3.32) that

$$
\begin{aligned}
\left(2^{2 r} s^{2}-\right. & 1)^{y / 2-1}=\left|\frac{\alpha^{z / 2}+\beta^{z / 2}}{\alpha+\beta}\right| \\
& =\left|\sum_{j=0}^{(z / 2-1) / 2}(-1)^{j}\left[\begin{array}{c}
z / 2 \\
j
\end{array}\right]\left(-2\left(2^{2 r} s^{2}-1\right)\right)^{z / 2-2 j-1}\left(2^{2 r} s^{2}+1\right)^{2 j}\right| \\
& =\left|\sum_{j=0}^{(z / 2-1) / 2}(-1)^{j}\left[\begin{array}{c}
z / 2 \\
(z / 2-1) / 2-j
\end{array}\right]\left(4\left(2^{2 r} s^{2}-1\right)^{2}\right)^{j}\left(2^{2 r} s^{2}+1\right)^{z / 2-2 j-1}\right| .
\end{aligned}
$$

If $y>2$, then we have

$$
y \geq 6
$$

by (3.24). Since

$$
\left[\begin{array}{c}
z / 2 \\
(z / 2-1) / 2
\end{array}\right]=\frac{z}{2}
$$


by (2.1), we see from (3.33), (3.34) and (3.35) that

$$
\frac{z}{2} \equiv 0 \quad\left(\bmod 2^{2 r} s^{2}-1\right) \text {. }
$$

Let $p$ be an odd prime divisor of $2^{2 r} s^{2}-1$, and let

$$
p^{\gamma}\left\|\frac{z}{2}, p^{\delta}\right\| 2^{2 r} s^{2}-1, p^{\delta_{j}} \| 2 j+1, j \geq 1 .
$$

Then we have

$$
\delta_{j} \leq \frac{\log (2 j+1)}{\log p} \leq j, j \geq 1
$$

By $(2.1)$, if $2 \nmid n$, then

$$
\left[\begin{array}{c}
n \\
(n-1) / 2-j
\end{array}\right]=n\left(\begin{array}{c}
(n-1) / 2+j \\
2 j
\end{array}\right) \frac{1}{2 j+1}, j=0, \cdots, \frac{n-1}{2} .
$$

Hence, by (3.35), (3.37),(3.38) and (3.39), we get

$$
p^{\gamma} \|\left[\begin{array}{c}
z / 2 \\
(z / 2-1) / 2
\end{array}\right]\left(2^{2 r} s^{2}+1\right)^{z / 2-1}
$$

and

$$
\begin{aligned}
& {\left[\begin{array}{c}
z / 2 \\
(z / 2-1) / 2-j
\end{array}\right]\left(4\left(2^{2 r} s^{2}+1\right)^{2}\right)^{j}\left(2^{2 r} s^{2}+1\right)^{z / 2-2 j-1}} \\
& \quad \equiv \frac{z}{2}\left(2^{2 r} s^{2}+1\right)^{z / 2-2 j-1}\left(\begin{array}{c}
(z / 2-1) / 2+j \\
2 j
\end{array}\right) \frac{4^{j}\left(2^{2 r} s^{2}-1\right)^{2 j}}{2 j+1} \\
& \quad \equiv 0 \quad\left(\bmod p^{\gamma+1}\right), j=1, \cdots, \frac{z / 2-1}{2} .
\end{aligned}
$$

Therefore, by (3.33), (3.37), (3.40) and (3.41), we obtain

$$
\left(\frac{y}{2}-1\right) \delta=\gamma .
$$

Taking $p$ through over all the distinct prime divisors of $2^{2 r} s^{2}-1$, by (3.37) and (3.42), we have

$$
\frac{z}{2} \equiv 0 \quad\left(\bmod \left(2^{2 r} s^{2}-1\right)^{y / 2-1}\right)
$$

whence we get

$$
\frac{z}{2} \geq\left(2^{2 r} s^{2}-1\right)^{y / 2-1}
$$

The combination of (3.21) and (3.43) yields

$$
y>\left(2^{2 r} s^{2}-1\right)^{y / 2-1} .
$$

But, by (3.34), (3.44) is false. So we have $y=2$.

Since $y=2$, by (3.18) and (3.21), we get $z=2$. Thus, by $(3.1),(x, y, z)=\left(2^{r+1} s, 2,2\right)$ is the unique solution of (1.3). The theorem is proved.

Acknowledgment. We would like to thank to referee for reading the paper carefully and her/his useful corrections. 


\section{References}

[1] M.A. Bennett, J.S. Ellenberg and N.C. Ng, The Diophantine equation $A^{4}+2^{\delta} B^{2}=$ $C^{n}$, Int. J. Number Theory, 6 (2), 311-338, 2010.

[2] X.G. Chen and M.H. Le, A note on Terai's conjecture concerning Pythagorean numbers, Proc. Japan Acad. Ser. A, 74 (5), 80-81, 1998.

[3] H.W. Gould, Tables of combinatorial identities, https://web.archive.org/web/ 20190629193344/http://www. math.wvu. edu/ gould/ (Gould's personal webpage).

[4] J.Y. Hu and H. Zhang, A conjecture concerning primitive Pythagorean triples, Int. J. Appl. Math. Stat. 52 (7), 38-42, 2014.

[5] M.H. Le, A note on the Diophantine equation $x^{2}+b^{y}=c^{z}$, Acta Arith. 71 (3), 253-257, 1995.

[6] M.H. Le, On Terai's conjecture concerning Pythagorean numbers, Acta Arith. 100 (1), 41-45, 2001.

[7] M.H. Le and G. Soydan, A brief survey on the generazlized Lebesgue-RamanujanNagell equation, Surv. Math. Appl. 15, 473-523, 2020.

[8] R. Lidl and H. Neiderreiter, Finite Fields, Cambridge Univ. Press, Cambridge, 1996.

[9] L.J. Mordell, Diophantine equations, Academic Press, London, 1969.

[10] G. Soydan, M. Demirci, I.N. Cangül and A. Togbé, On the conjecture of Jeśmanowicz, Int. J. Appl. Math. Stat. 56 (6), 46-72, 2017.

[11] N. Terai, A note on the Diophantine equation $x^{2}+q^{m}=p^{n}$, Acta Arith. 63 (4), 351-358, 1993.

[12] P.Z. Yuan, The Diophantine equation $x^{2}+b^{y}=c^{z}$, J. Sichuan Univ. Nat. Sci. 41 525-530, 1998 (in Chinese).

[13] P.Z. Yuan and J.B. Wang, On the Diophantine equation $x^{2}+b^{y}=c^{z}$, Acta Arith. 84 (2), 145-147, 1998. 